\title{
Erlotinib inhibits osteolytic bone invasion of human non-small-cell lung cancer cell line NCI-H292
}

\author{
Koh Furugaki · Yoichiro Moriya · Toshiki Iwai • \\ Keigo Yorozu • Mieko Yanagisawa $\cdot$ Kumiko Kondoh • \\ Kaori Fujimoto-Ohuchi $\cdot$ Kazushige Mori
}

Received: 5 January 2011 / Accepted: 31 May 2011/Published online: 18 June 2011

(C) The Author(s) 2011. This article is published with open access at Springerlink.com

\begin{abstract}
Previous preclinical and clinical findings have suggested a potential role of epidermal growth factor receptor (EGFR) in osteoclast differentiation and the pathogenesis of bone metastasis in cancer. In this study, we investigated the effect of erlotinib, an orally active EGFR tyrosine kinase inhibitor (TKI), on the bone invasion of human non-small-cell lung cancer (NSCLC) cell line NCI-H292. First, we established a novel osteolytic bone invasion model of NCI-H292 cells which was made by inoculating cancer cells into the tibia of scid mice. In this model, NCI-H292 cells markedly activated osteoclasts in tibia, which resulted in osteolytic bone destruction. Erlotinib treatment suppressed osteoclast activation to the basal level through suppressing receptor activator of NF- $\kappa \mathrm{B}$ ligand (RANKL) expression in osteoblast/stromal cell at the bone metastatic sites, which leads to inhibition of osteolytic bone destruction caused by NCI-H292 cells. Erlotinib inhibited the proliferation of NCI-H292 cells in in vitro. Erlotinib suppressed the production of osteolytic factors, such as parathyroid hormone-related protein (PTHrP), IL-8, IL-11 and vascular endothelial growth factor (VEGF) in NCI-H292 cells. Furthermore, erlotinib also inhibited osteoblast/stromal cell proliferation in vitro and the development of osteoclasts induced by RANKL in vitro. In conclusion, erlotinib inhibits tumor-induced osteolytic invasion in bone metastasis by suppressing osteoclast
\end{abstract}

K. Furugaki · Y. Moriya $(\varangle) \cdot$ T. Iwai $\cdot$ K. Yorozu

M. Yanagisawa $\cdot$ K. Kondoh $\cdot$ K. Fujimoto-Ohuchi $\cdot$ K. Mori

Product Research Department, Kamakura Research

Laboratories, Chugai Pharmaceutical Co., Ltd., 200 Kajiwara,

Kamakura, Kanagawa 247-8530, Japan

e-mail: moriyayui@chugai-pharm.co.jp activation through inhibiting tumor growth at the bone metastatic sites, osteolytic factor production in tumor cells, osteoblast/stromal cell proliferation and osteoclast differentiation from mouse bone marrow cells.

Keywords Epidermal growth factor receptor - Tyrosine kinase inhibitor - Osteolytic bone invasion model .

Osteoclast

$\begin{array}{ll}\text { Abbreviation } \\ \text { EGFR } & \text { Epidermal growth factor receptor } \\ \text { NSCLC } & \text { Non-small-cell lung cancer } \\ \text { PTHrP } & \text { Parathyroid hormone-related protein } \\ \text { VEGF } & \text { Vascular endothelial growth factor } \\ \text { SRE } & \text { Skeletal-related event } \\ \text { TKI } & \text { Tyrosine kinase inhibitor } \\ \text { MEM } & \text { Minimum essential medium } \\ \text { HE } & \text { Hematoxylin and eosin } \\ \text { TRAP } & \text { Tartrate-resistant acid phosphatase } \\ \text { ALP } & \text { Alkaline phosphatase } \\ \text { OPG } & \text { Osteoprotegerin } \\ \text { RANKL } & \text { Receptor activator of NF- } \kappa \text { B ligand }\end{array}$

\section{Introduction}

Non-small-cell lung cancer (NSCLC) is the most common type of lung cancer, accounting for approximately $80 \%$ of lung cancers. Bone metastases occur in 30-40\% of NSCLC patients, and are often associated with significant morbidity [1]. Common bone metastatic complications, which are also called skeletal-related events (SREs), include bone pain, pathological fractures, malignant hypercalcemia and spinal cord compression. These SREs are clinically meaningful 
sequelae that are associated with health care cost and decreased quality of life. Zoledronic acid is one of the widely used bisphosphonates that has been demonstrated to significantly prevent SREs in lung cancer patients with bone metastases. However, there are no significant differences in time of bone-lesion progression and median survival between zoledronic acid and a placebo [2]. Thus, it is necessary to develop an effective therapy that could inhibit the tumor growth and extend survival in NSCLC patients with bone metastases.

Erlotinib is an oral, small-molecule epidermal growth factor receptor tyrosine kinase inhibitor (EGFR-TKI) that reversibly binds to the intracellular tyrosine kinase domain of EGFR and is used as a standard treatment for previously-treated advanced NSCLC. The expression of EGFR and its ligand has been found in the majority of human carcinoma. Erlotinib blocks autophosphorylation of EGFR with subsequent inhibition of the downstream signaling cascades involving RAS/RAF/MAPK and PI3K/ AKT. The EGFR signaling network, one of important process involved in tumor progression affecting cell proliferation, inhibition of apoptosis, metastasis and angiogenesis, is often dysregulated in cancer cells [3]. Erlotinib monotherapy has been favorable to the presence of EGFR mutations and both EGFR and KRAS wild-types in NSCLC [4].

In clinical studies, treatment with EGFR-TKI, erlotinib or gefitinib, for NSCLC patients with bone metastases was reported to significantly decrease bone pain, to improve pathologic fractures, to prolong disease control of bone metastatic site and also to significantly extend overall survival [5-9]. Although, these clinical observations indicate the possibility of EGFR signal involvement in the progression of bone metastases of NSCLC, there are no prospective, large size clinical studies.

In preclinical studies, various activities of EGFR-TKI on the pathogenesis and progression of bone metastases of prostate cancer and renal cancer have been described in previous reports [10-12]. Normanno et al. [13] described that gefitinib has a potential in bone metastases by inhibiting the ability of human bone marrow stromal cells to induce osteoclast differentiation. Moreover, a number of different mechanisms have been suggested to be involved in these effects [14]. However there has been no report to show an activity of EGFR-TKI on bone metastases of NSCLC cells.

In this study, for evaluating whether or not erlotinib would be effective for bone metastases of NSCLC, we tried to establish a novel osteolytic bone invasion model which resembles the clinical characteristics of NSCLC. Then we examined whether erlotinib could be a useful agent for bone metastases of NSCLC using our novel osteolytic bone invasion model.

\section{Materials and methods}

Chemicals

Erlotinib was provided by F. Hoffman-La Roche Ltd. (Basel, Switzerland). Erlotinib was dissolved in DMSO for the in vitro assay and in $6 \% \mathrm{Captisol}^{\circledR}$ (CyDex Pharmaceuticals, Inc., Lenexa, KS) solution for the in vivo experiment. Captisol was dissolved in distillated water.

\section{Cell lines}

Human NSCLC cell line NCI-H292, human osteoblast cell line MG-63 and human stromal cell line HS-5 were purchased from the American Type Culture Collection (ATCC, Manassas, VA) and maintained in RPMI1640 (Sigma-Aldrich Co., Ltd., St. Louis, MO) supplemented with 10\% FBS (Japan Bio Serum Co., Ltd., Fukuyama, Japan). Mouse osteoblastic cell line MC3T3-E1 and mouse stromal cell line ST2 were purchased from RIKEN BioResource Center (Tsukuba, Japan) and maintained in aMEM (Invitrogen, Carlsbad, CA) and in RPMI1640 supplemented with $10 \%$ FBS, respectively. Mouse primary osteoblasts were purchased from Primary Cell Co., Ltd. (Sapporo, Japan) and maintained in $\alpha \mathrm{MEM}$ supplemented with $10 \%$ FBS. All cells were cultured at $37^{\circ} \mathrm{C}$ in $5 \% \mathrm{CO}_{2}$.

\section{Animals}

Male 5-week-old ddY mice, BALB-nu/nu mice and scid mice were purchased from Japan SLC, Inc. (Hamamatsu, Japan), Charles River Japan, Inc. (Yokohama, Japan) and Clea Japan, Inc. (Osaka, Japan), respectively. Animals were housed in a pathogen-free environment under controlled conditions (temperature: $20-26^{\circ} \mathrm{C}$, humidity: 40-70\%, light-dark cycle: 12-12 h). Chlorinated water and irradiated food were provided ad libitum. The animals were allowed to acclimatize and recover from shipping-related stress for more than 7 days prior to the study. The health of the mice was monitored daily. All animal protocols were reviewed by the Institutional Animal Care and Use Committee of Chugai Pharmaceutical Co., Ltd and all animal experiments were performed in accordance with the 'Guidelines for the Accommodation and Care of Laboratory Animals' of Chugai Pharmaceutical Co., Ltd.

Intratibial osteolytic bone invasion model of NCI-H292 cells

Tibial implantation of NCI-H292 cells was performed as described [15]. Briefly, $2 \times 10^{5}$ NCI-H292 cells in $20 \mu \mathrm{l}$ of culture medium/matrigel (BD Biosciences, San Jose, CA) 
mix were injected into both tibiae and the mice were randomly divided into two groups of 10 mice on day 1 . The mice were administered once daily either oral erlotinib $(75 \mathrm{mg} / \mathrm{kg} / \mathrm{day})$ or Captisol, as a vehicle control, starting from day 1 for 27 days. The mice were also subjected to deep anaesthesia and exposed to soft X-rays at $30 \mathrm{kV}$ for $2 \mathrm{~s}$ with SOFRON (SOFRON Co., Ltd., Tokyo, Japan) on days 14, 21 and 28 as described [16]. The radiophotographs were scanned with a digital camera (Nicon, Tokyo, Japan), and the number and the area of osteolytic foci of bone metastases in the whole tibia were measured using an image analyzing software (WinROOF, Mitani Corporation, Fukui, Japan).

Histologic and histomorphometric analysis of osteolytic lesions

The tibias of mice treated daily with either erlotinib or vehicle from day 1 to day 13 were collected on day 14 , and fixed in $4 \%$ paraformaldehyde. The specimens were decalcified in EDTA and embedded in paraffin. Thin sections were stained with hematoxylin and eosin (HE) staining, tartrate-resistant acid phosphatase (TRAP) staining. Immunohistochemical stainings were also performed using anti-mouse receptor activator of NF- $\kappa \mathrm{B}$ ligand (RANKL) antibody (R\&D systems, Minneapolis, MN), anti-phospho-EGFR (Y1068) antibody or anti-Ki-67 antibody (Abcam, Cambridge) as described [17] and according to the manufacturer's instructions. The tibias were collected on day 14, and fixed in $70 \%$ ethanol. The specimens were embedded in glycolmethacrylate. Thin sections were stained with alkaline phosphatase (ALP)/TRAP double staining. Histomorphometric analysis of the tumor burden, tumor proliferation and number of osteoclasts in tibia was performed using the proximal sections stained with HE, Ki-67 and TRAP, respectively. The tumor area was measured using image analyzing software (Scion, Frederick, MD). The number of activated osteoclasts was determined by counting the multinucleated ( $>4$ nuclei) TRAP-positive cells under a light microscope.

\section{Immunoblotting}

Immunoblotting was performed as described [18]. Briefly, cultured cells were lysed in lysis buffer and the $20 \mu \mathrm{g}$ protein lysates were separated on 7.5\% SDS-PAGE and then transferred onto the membrane. Antibodies against EGFR, phospho-EGFR (Y1068), ERK, phospho-ERK, AKT, phospho-AKT (S473) (Cell Signaling Technology, Inc., Danvers, MA) and anti- $\beta$-actin (Sigma-Aldrich) were used for immunoblotting.
In vitro cell proliferation assay

Cells were seeded $1 \times 10^{3}$ cells/well in 96-well plates. From the following day, the cells were treated with erlotinib for 4 days and then MTT assays were done using WST-8 solution (Dojindo, Tokyo, Japan) according to the manufacturer's instructions. The viability of cells was calculated as: [(mean absorbance of erlotinib-treated wells - mean absorbance of cell-free wells)/(mean absorbance of vehicle wells - mean absorbance of cell-free wells)] $\times$ 100.

Coculture of NCI-H292 cells and mouse primary osteoblasts or stromal cells

To evaluate interaction between these cells, three coculture conditions were performed as described previously [18]. Briefly, $1 \times 10^{4}$ mouse primary osteoblasts or stromal cell line ST2 were cocultured with NCI-H292 confluent cell monolayer with or without $3 \%$ paraformaldehyde fixation. Or $1 \times 10^{4}$ mouse primary osteoblasts/ST2 cells were cocultured with $1 \times 10^{5} \mathrm{NCI}-\mathrm{H} 292$ cells in suspension with or without a an $8.0 \mu \mathrm{m}$ pore size cell culture insert (Corning, Inc., Lowell, MA) to separate from each cell. After $8 \mathrm{~h}$ coculture, cDNA of primary osteoblasts and ST2 were synthesized using the Cells-to-cDNA kit (Ambion, Inc., Austin, TX), and the real-time RT-PCR was performed to examine the mouse RANKL, osteoprotegerin (OPG) and actin mRNA expression level using LightCycler system (Roche Diagnostics, Ltd., Basel, Switzerland). Applied Biosystems Assay-on-Demand primer probe sets for the detection of mouse RANKL, OPG and actin were used with Mm00441908m1, Mm00446190m1 and Mm00435452m1, respectively (Life Technologies Co., Ltd., Carlsbad, CA). Relative gene expression compared to non-treated cells was quantified using the comparative threshold cycle $\left(\mathrm{C}_{\mathrm{T}}\right)$ method by subtracting the internal control of actin $C_{T}$ value from the RANKL or OPG $C_{T}$ value. These $C_{T}$ values were calculated by the 2 nd derivative maximum method in LightCycler system.

In vitro production of osteolytic factors in NCI-H292 cells

The $2 \times 10^{6}$ NCI-H292 cells were seeded in 6-well plates. On the following day, cells were treated with erlotinib for 1 day and then protein levels of osteolytic factors (parathyroid hormone-related protein (PTHrP), IL-1 $\beta, 6,8,10$, $11, \mathrm{GM}-\mathrm{CSF}, \mathrm{TNF}-\alpha$ and vascular endothelial growth factor (VEGF)) [19-21] in the culture media and the cellular DNA contents were determined by immunoassays and a double-strand DNA Quantification Kit (Invitrogen). PTHrP and IL-11 were measured by IRMA in Mitsubishi 
Chemical Medience Corporation (Tokyo, Japan) and ELISA kit (R\&D systems), respectively. The others were measured by Bio-plex human cytokine assays (Bio-Rad Laboratories, Inc., Hercules, CA). The protein level of osteolytic factors normalized by the DNA contents were calculated as: [(concentration of erlotinib-treated wells - mean concentration of cell-free wells)/DNA contents of erlotinib-treated wells]/[(concentration of vehicle-treated wells - mean concentration of cell-free wells)/DNA contents of vehicle-treated wells] $\times 100$. The data were shown as mean $+\mathrm{SD}$ of duplicates.

In vitro osteoclast differentiation from mouse bone marrow cells

The protocol was performed as described [22]. Briefly, mouse bone marrow cells were isolated from 6 to 10 -week-old male ddY mice and then cultured in $\alpha \mathrm{MEM}$ supplemented with $10 \% \mathrm{FBS}, 30 \mathrm{ng} / \mathrm{ml}$ mouse M-CSF (Wako Pure Chemical Industries, Ltd., Osaka, Japan) and $30 \mathrm{ng} / \mathrm{ml}$ mouse RANKL (Wako) with or without erlotinib. After osteoclast differentiation, cells were fixed and stained with TRAP. The number of osteoclasts was determined by counting the multinucleated ( $>3$ nuclei) TRAP-positive cells under a microscope. The data were shown as mean + $\mathrm{SD}$ of quadruplicates.

\section{Statistical analysis}

The Wilcoxon test was used to detect the statistical differences in tumor volume. Probability values $<0.05$ were considered to be significant. The $t$ test was used to detect the statistical differences in number of osteoclasts. Probability values $<0.05$ were considered to be significant. Statistical analyses were performed using an SAS preclinical package (version 8.2; SAS Institute, Inc., Cary, NC).

\section{Results}

Establishment of mouse osteolytic bone invasion model of NCI-H292 cells

To examine the effects of erlotinib on bone metastasis of NSCLC, we established a novel in vivo intratibial osteolytic bone invasion model of the human NSCLC cell line NCI-H292. In mice injected with NCI-H292 cells into tibia, osteolytic bone lesions appeared from day 14 and expanded in a time-dependent manner (Fig. 1a, b). The tumor take rate in this model was $90 \%$ (osteolytic bone lesions were observed only in eighteen of twenty legs inoculated on day 28). ALP/TRAP double staining demonstrated that many TRAP-positive activated osteoclasts were present within the ALP-positive osteoblast/stromal cell layer covering the bone surfaces close to the metastatic tumor (Fig. 1c). In contrast, activated osteoclasts could hardly be seen in normal mouse (Fig. 1c). These findings indicate that direct contact between NCI-H292 tumor cells and osteoblasts/stromal cells induced the activation of osteoclastic precursors in the metastatic lesions.

NCI-H292 cell-induced RANKL expression in mouse osteoblasts/stromal cells ST2

Osteoclasts are developed by cellular interaction between osteoclastic precursors and osteoblasts or stromal cells thorough RANK-RANKL signal transduction [23]. To examine the mechanism of NCI-H292 cell-induced osteoclast activation in this model, we investigated the effects of NCI-H292 cells on RANKL expression in mouse primary osteoblasts and mouse stromal cells ST2. The expression of RANKL mRNA in osteoblasts/ST2 cells was drastically increased when cultured with live adherent NCI-H292 cells, but were strongly abolished when cultured with fixed tumor cells (Fig. 1d). Furthermore, RANKL mRNA expression in osteoblasts/stromal cells was also drastically increased when cultured with NCI-H292 cells in suspension, but were strongly abolished when cultured apart from tumor cells (Fig. 1d). In contrast, there was almost no change in OPG expression in osteoblasts/stromal cells when these were cocultured with adhesion and suspension of NCI-H292 cells (data not shown). These findings indicate that not only cell-to-cell direct contact but also any soluble factors from tumor cells are indispensable for NCIH292 cell-induced RANKL expression in osteoblasts/ stromal cells.

Inhibitory effects of erlotinib on osteolytic bone invasion model of NCI-H292 cells

Administration of erlotinib significantly decreased the area and incidences of NCI-H292 cell-induced osteolytic lesions on days 21 and 28 (Fig. 2a, b, c). Administration of erlotinib starting from days 7 or 14 exerted almost the same inhibitory effects of osteolytic lesions on day 28 as starting from day 1 (data not shown). A difference in body weight loss was not observed in the erlotinib treatment group compared with the vehicle group on days 21 and 28 (data not shown). Furthermore, erlotinib completely suppressed the tumor induced-RANKL expression in osteoblasts/stromal cells (Fig. 2d). Erlotinib markedly suppressed the osteoclast activation to the basal level of non-tumor bearing mouse $(P=0.07)$ (Fig. 2e, f). These findings indicate that erlotinib inhibited osteolytic bone destruction of NCIH292 cells in the tibia by inhibiting the accumulation of activated osteoclasts. 
Fig. 1 NCI-H292 cells induced osteolytic lesions in tibia by activating osteoclasts through the interaction between osteoblasts/stromal cells and NCI-H292 cells.

a Radiophotograph. Arrowheads indicate the osteolytic foci. Arrowheads indicate the osteolytic foci. b The total area of osteolytic foci. Each point represents the mean + SE of 20

determinations. c Histological pictures of non-tumor-bearing mouse and NCI-H292 tumorbearing mouse double stained for TRAP/ALP on day 13 .

TRAP-positive activated osteoclasts (red, OC). ALPpositive osteoblasts/stromal cell layer (purple, ST). d Mouse

RANKL expression in osteoblasts/stromal cells cocultured with NCI-H292 confluent cell monolayer, and with NCI-H292 cells in suspension. Each point represents the mean $+\mathrm{SD}$ of duplicates
A

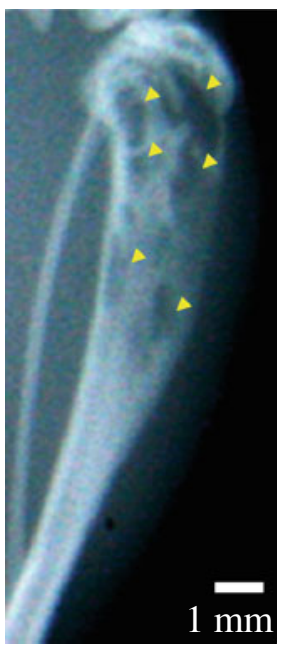

Day 28

D
B

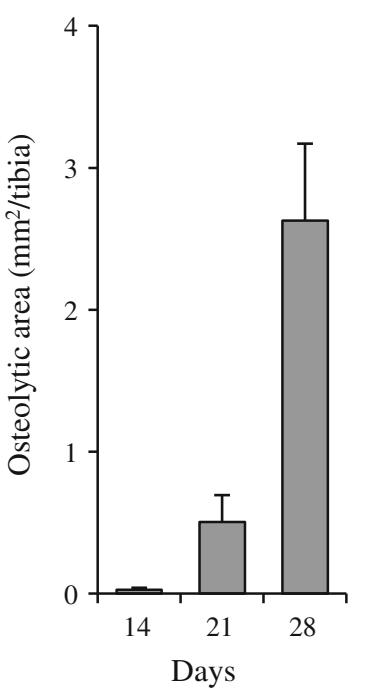

C

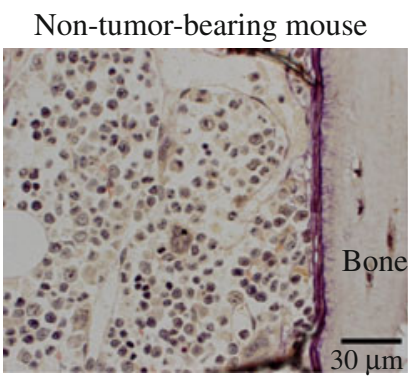

Tumor-bearing mouse

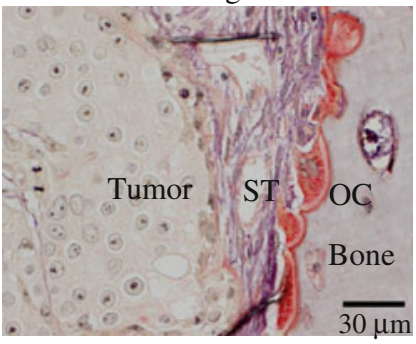

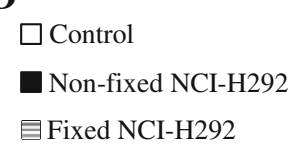

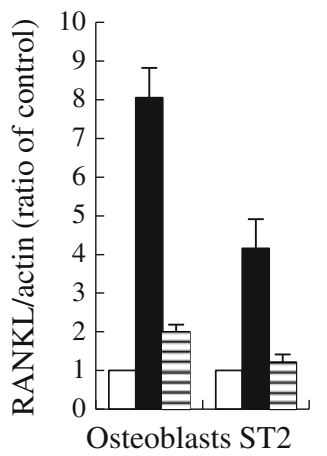

$\square$ Control

without culture insert

目 with culture insert

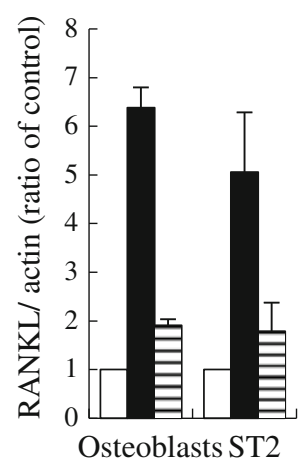

Inhibitory effects of erlotinib on cell proliferation of NCI-H292 cells in vivo and in vitro

Administration of erlotinib tended to inhibit the metastatic tumor growth in this osteolytic bone invasion model $(P=0.24)$ (Fig. 3a, b). The reduced cell proliferative activity of NCI-H292 cells was observed by immunostaining of proliferative markers Ki-67 $(P=0.24)$ (Fig. 3c). Furthermore, erlotinib showed a marked inhibition of NCI-H292 cell proliferation in vitro in a dosedependent manner, and the $\mathrm{IC}_{50}$ value was $0.11 \mu \mathrm{M}$ (Fig. 3d). An addition of $1 \mu \mathrm{M}$ of erlotinib markedly blocked not only EGFR phosphorylation but also ERK and Akt phosphorylation which are the downstream of EGFR signaling cascades in NCI-H292 cells (Fig. 3e). Meanwhile, phosphorylated EGFR expression in tumor cells at the metastatic sites was detected in not only the vehicle treatment but also the erlotinib treatment, a difference of the level of EGFR phosphorylation was not observed with or without erlotinib treatment (Fig. 3f).

Inhibitory effects of erlotinib on production of osteolytic factor in NCI-H292 cells

To elucidate the soluble factor from NCI-H292 cells which is related to RANKL expression in osteoblasts/ST2 cells, we investigated the production of major osteolytic factors including PTHrP, IL-6 and IL-8 etc. As a result, NCI-H292 cells produced a sufficient amount of PTHrP, IL-6, IL-8, IL-11 and VEGF but the production of IL- $1 \beta$, IL-10, GM-CSF and TNF- $\alpha$ was lower than the detection limit (data not shown). Erlotinib blocked the production of PTHrP, IL-8, IL-11 and VEGF in NCI-H292 cells in a dose-dependent manner (Fig. 4). Meanwhile, IL-6 production was not changed by erlotinib treatment, indicating that IL-6 expression is independent of the EGFR signal in NCI-H292 cells (Fig. 4). 
A

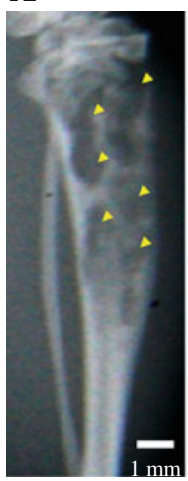

Vehicle
B

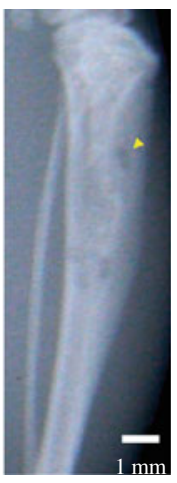

Erlotinib

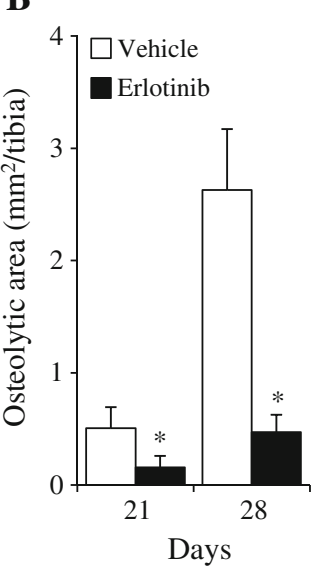

C

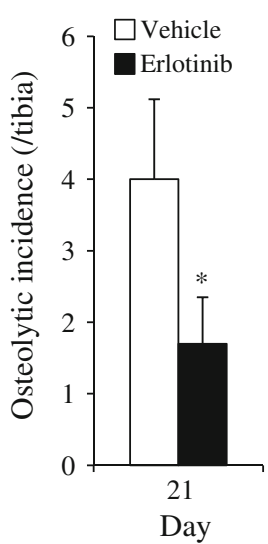

F

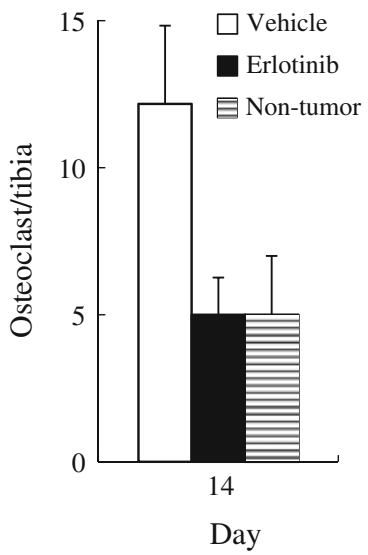

D

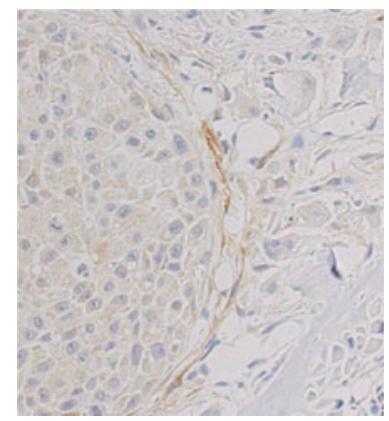

Vehicle

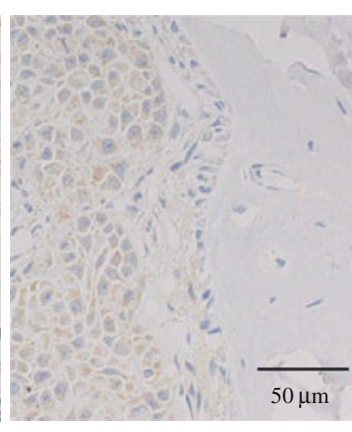

Erlotinib
Fig. 2 Effects of erlotinib on osteolytic bone invasion model of NCIH292 cells. NCI-H292 cell-induced osteolytic lesions in mice treated with or without $75 \mathrm{mg} / \mathrm{kg}$ of erlotinib. a Radiophotographs on day 28 . Arrowheads indicate the osteolytic foci. The total area (b) and incidence $(\mathbf{c})$ of osteolytic foci were measured. Each point represents the mean $+\mathrm{SE}$ of 20 determinations. $* P<0.05$, by Wilcoxon test, compared with vehicle-treated group. d Histological pictures on day

Inhibitory effects of erlotinib on cell proliferation of osteoblasts/stromal cells and osteoclast differentiation from mouse bone marrow cells

Recently, the role of EGFR in osteoblast and osteoclast has been focused on [5, 24]. Therefore, we examined the effects of erlotinib on these cells. The mouse primary osteoblasts, mouse osteoblastic cell line MC3T3-E1, mouse stromal cell line ST2, human osteoblastic cell line MG-63 and human stromal cell line HS-5 expressed EGFR (Fig. 5a). Erlotinib inhibited the proliferation of these cell lines in vitro (Fig. 5b, c). In contrast, the reduced cell proliferative activity of osteoblasts/stromal cells in vivo by erlotinib treatment could not be estimated, because there was no detectable Ki-67 immunostaining in osteoblasts/ stromal cells with or without erlotinib treatment (data not shown). In addition, erlotinib completely suppressed the formation of mononuclear and multinuclear TRAP-positive
$\mathbf{E}$

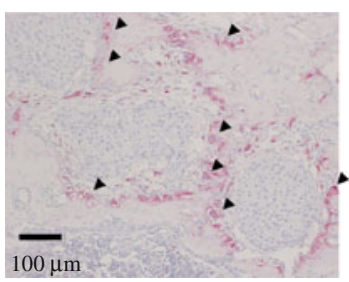

Vehicle

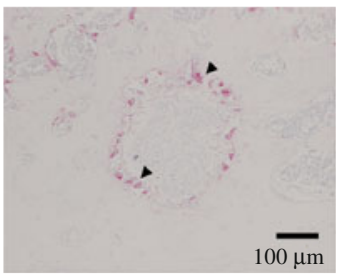

Erlotinib
14 stained with anti-mouse RANKL antibody. The stained area (brown) indicates the expression of RANKL in osteoblasts/stromal cells. e Histological pictures on day 14 stained with TRAP in mice treated with or without $75 \mathrm{mg} / \mathrm{kg}$ of erlotinib. Arrowheads indicate the activated osteoclasts. $\mathbf{f}$ The number of multinucleated ( $>4$ nuclei) TRAP-positive osteoclasts were measured. Each point represents the mean + SE of sex tuplicates

cells in a dose-dependent manner (Fig. 5d, e), although erlotinib did not exhibit any cytotoxity against bone marrow cells in this condition (data not shown).

\section{Discussion}

Bone is one of the commonest target organs of distant metastasis in lung cancer [25]. Many steps are involved in the metastasis from primary site to bone; that is, new vessel formation, invasion, arrest in secondary capillary beds, extravasation, adaptation to bone microenvironment and proliferation of cancer cells in bone [26, 27]. The main mechanism responsible for bone destruction is considered to be bone resorption, caused not directly by tumor cells but by tumor cell-activated osteoclasts through interaction with osteoblasts/stromal cells, which altogether form a "vicious circle" [28]. In the present study, we investigated 


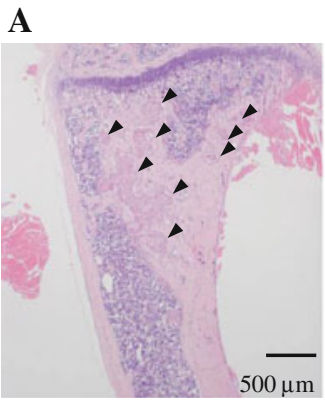

Vehicle

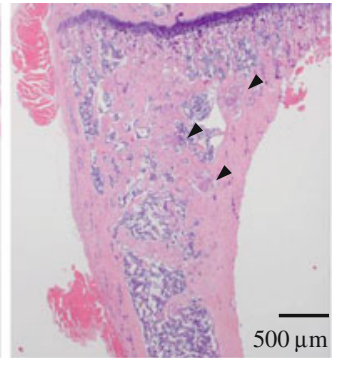

Erlotinib

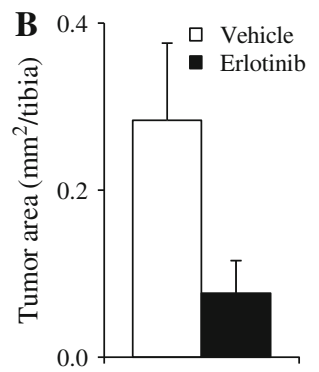

14

Day

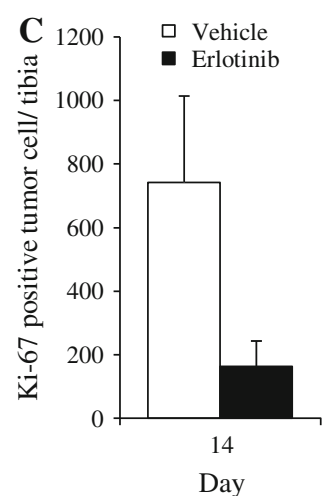

Erlotinib $(\mu \mathrm{M})$

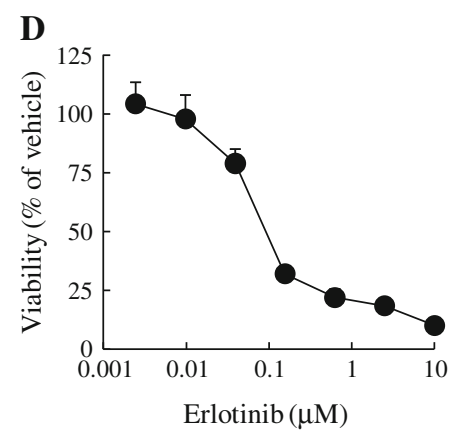

Fig. 3 Effects of erlotinib on cell proliferation of NCI-H292 cells. a NCI-H292 tumor cells grew in tibia of mice treated with or without $75 \mathrm{mg} / \mathrm{kg}$ of erlotinib. Histological pictures on day 14 stained with HE. Arrowheads indicate the metastatic tumor area. b The metastatic tumor area were measured. Each point represents the mean + SE of sex tuplicates. c The number of Ki-67-positive tumor cells was

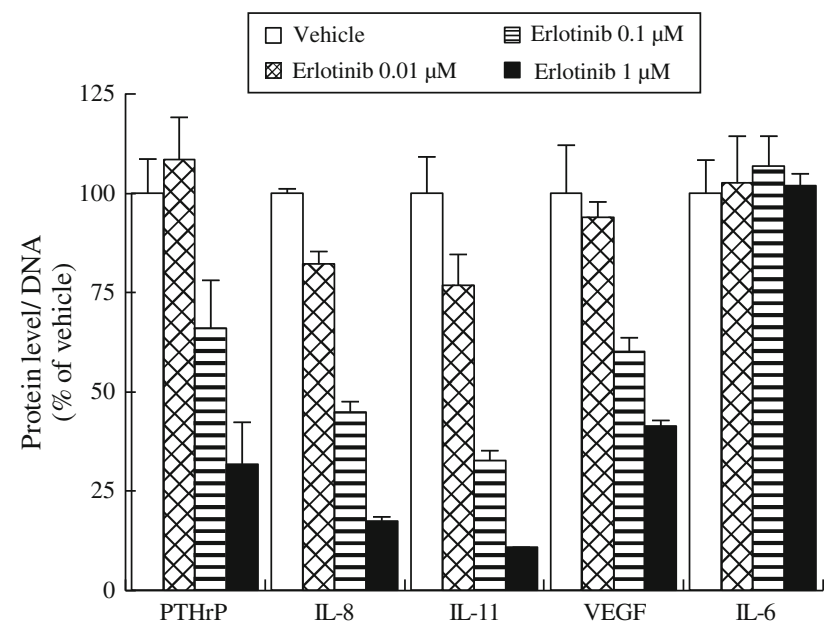

Fig. 4 Effects of erlotinib on production of osteolytic factor of NCIH292 cells. The protein levels of osteolytic factors in the conditioned media of NCI-H292 cells treated with or without $1 \mu \mathrm{M}$ erlotinib were measured. Each point represents the mean + SD of duplicates

the role of EGFR in the vicious circle of NSCLC bone metastasis by examining whether EGFR-TKI has the potential to inhibit bone metastases of NSCLC cells.

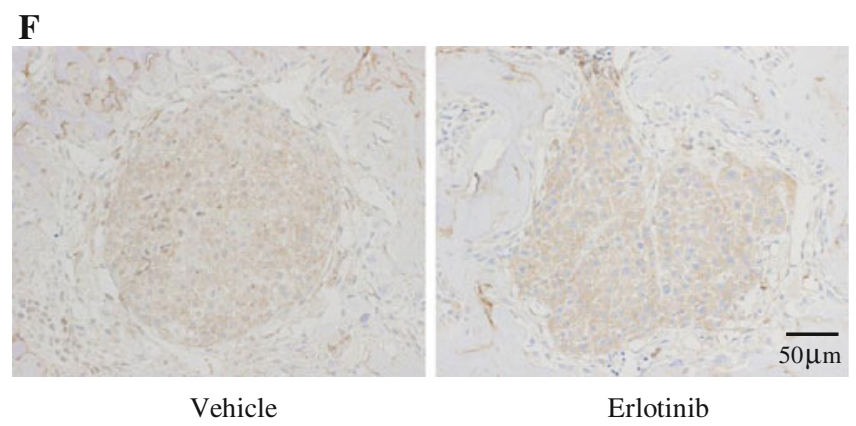

counted. Each point represents the mean + SE of sex tuplicates. d In vitro cell proliferation determined by MTT assay after 4 days of treatment with erlotinib. Each point represents the mean + SD of triplicates. e Immunoblots of cell lysates from cells treated with or without erlotinib. f Histological pictures on day 14 stained with phosphorylated EGFR

Although the effects of EGFR-TKI on bone metastases in NSCLC patients have not been investigated in a large clinical study, occasional reports have suggested a potential activity of this drug on the progression of bone disease [5-9]. Recently, Okano et al. [6] have reported cases of NSCLC in which gefitinib, an EGFR-TKI, showed a dramatic response to pathological fractures due to bone metastases and eventually the fractures were almost completely healed. Moreover, gefitinib showed a significant improvement of metastatic bone pain in breast cancer [29]. However, the impact of EGFR-TKI on bone metastases seems to be different in each patient.

To evaluate the potential of erlotinib on bone metastases in preclinical study, we tried to establish a novel bone invasion model using NSCLC cell line NCI-H292. Because the experimental bone metastasis models of NSCLC cells were very limited. NCI-H292 cells carry wild-types of both EGFR and KRAS, which are the most major characteristics $(60-76 \%)$ in all NSCLC patients $[30,31]$. This is the first osteolytic bone invasion model using a NSCLC cell line with both EGFR and KRAS wild-types. In this model, our histological studies showed that osteolytic bone destruction induced by NCI-H292 cells is caused by activated 

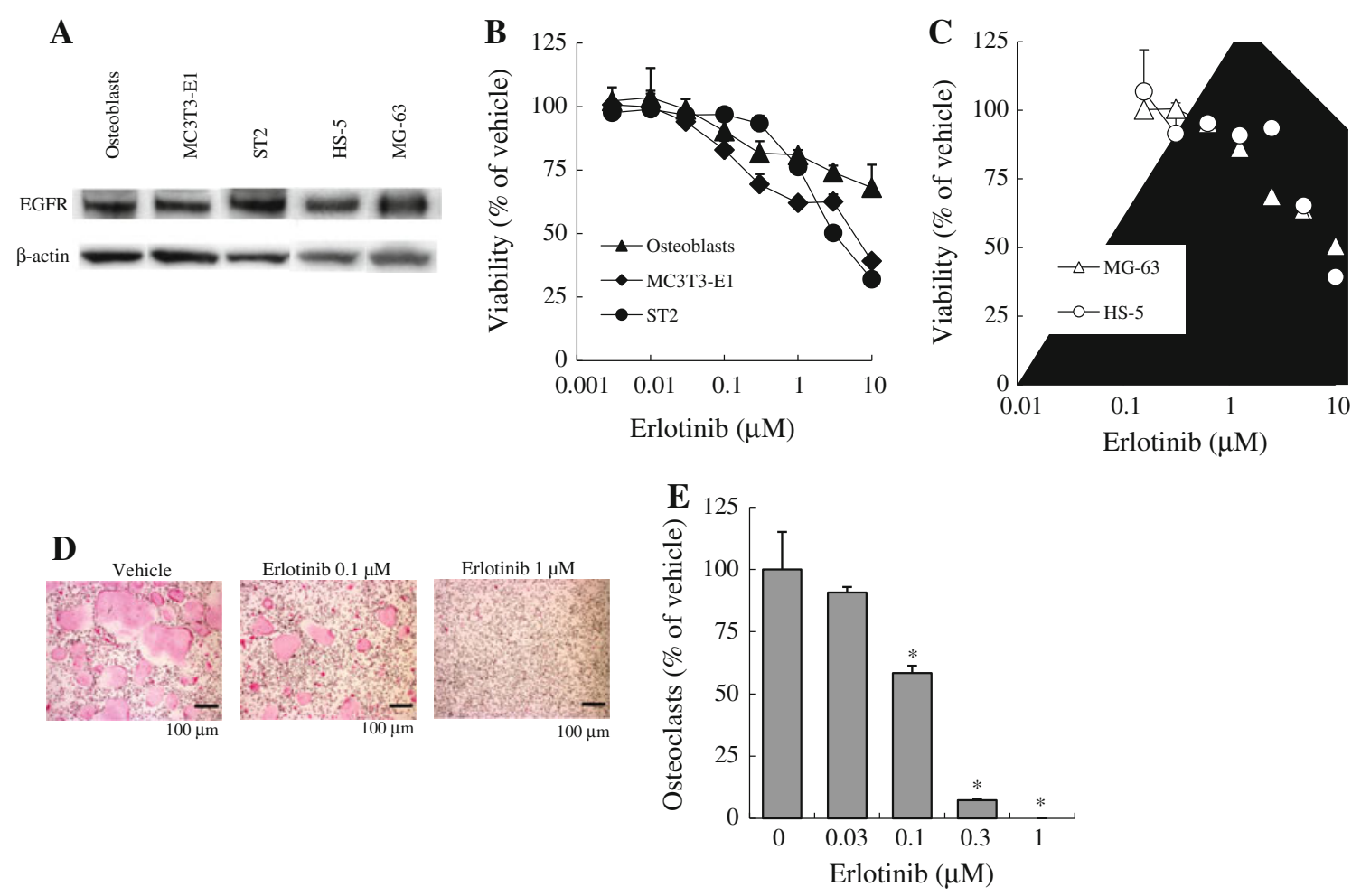

Fig. 5 Effects of erlotinib on cell proliferation of mouse and human osteoblasts/stromal cells and osteoclast differentiation from mouse bone marrow cells. a EGFR expression detected by immunoblotting with anti-EGFR antibody. $\mathbf{b}$ and $\mathbf{c}$ Cell proliferation was determined by MTT assay after 4 days of treatment with erlotinib. Each point represents mean $+\mathrm{SD}$ of triplicates. d Mouse bone marrow cells

osteoclast accumulation in the metastatic lesions through the interaction between NCI-H292 tumor cells and osteoblasts/stromal cells (Fig. 1). Furthermore, RANKL expression in osteoblasts/stromal cells caused by NCIH292 cells was indispensable not only for cell-to-cell contact but also some soluble factors, such as osteolytic factors, from NCI-H292 tumor cells (Fig. 1). RANKL is a critical osteoclast differentiation factor highly expressed on the membranes of the osteoblasts/stromal cells in the bone marrow environment [23]. RANKL combines with RANK, a receptor of RANKL expressed in osteoclastic precursors, and delivers intracellular signals involved in osteoclast differentiation [19]. At the site of bone metastases, RANKL is also considered as a direct mediator of tumorassociated osteolytic lesions [32]. These findings indicate that a vicious circle is formed in NCI-H292 osteolytic bone invasion model.

The molecules involved in cell-to-cell recognition between NCI-H292 cells and osteoblasts/stromal cells are unclear. Integrins are reported to be candidates. Previous reports have shown that osteoblasts as well as tumor cells express various integrins, such as $\beta_{1}$ integrin and $\alpha_{\mathrm{v}} \beta_{3}$ integrin, and integrin signals regulate osteoclast formation were cultured in osteoclast differentiation medium with or without erlotinib for 12 days. Micrographs of TRAP staining. e The number of multinucleated ( $>3$ nuclei) TRAP-positive osteoclasts was measured. Each point represents mean $+\mathrm{SD}$ of quadruplicates. $* P<0.05$, by $t$ test, compared with vehicle-treated group

$[33,34]$. However, we do not investigate whether or not integrins are involved in interaction between NCI-H292 cells and osteoblasts/stromal cells, further studies are needed to identify the molecules.

In the present study, erlotinib treatment suppressed osteoclast activation, which led to inhibit osteolytic bone destruction caused by NCI-H292 cells (Fig. 2). To clarify the bone metastasis inhibitory mechanism of erlotinib would be a useful approach to clinically select NSCLC patients with bone metastases who could benefit from erlotinib treatment. We demonstrated that erlotinib affects the vicious circle of NCI-H292 osteolytic bone invasion which results in inhibition of osteoclast activation. The effects of erlotinib appear to be related to four different mechanisms: the suppression of (1) tumor growth at the bone metastatic sites, (2) osteolytic factor production, such as PTHrP, IL-6, IL-8, IL-11 and VEGF in tumor cells, (3) osteoblasts/ stromal cell proliferation and (4) osteoclast differentiation from mouse bone marrow cells (Fig. 6).

First, erlotinib strongly inhibited the proliferation of NCI-H292 cells in vitro by blocking EGFR phosphorylation with subsequent inhibition of the downstream signaling cascades involving RAS/RAF/MAPK and PI3K/AKT 
Fig. 6 A schematic diagram of the mechanism of erlotinib in a NCI-H292 osteolytic bone invasion model. Erlotinib suppressed NCI-H292 cellinduced osteoclast activation by suppressing metastatic tumor growth, osteolytic factor production in tumor cells, osteoblasts/stromal cell proliferation and osteoclast differentiation from mouse bone marrow cells. Abbreviations: $O B / S T$ osteoblast/stromal, $O C$ osteoclast

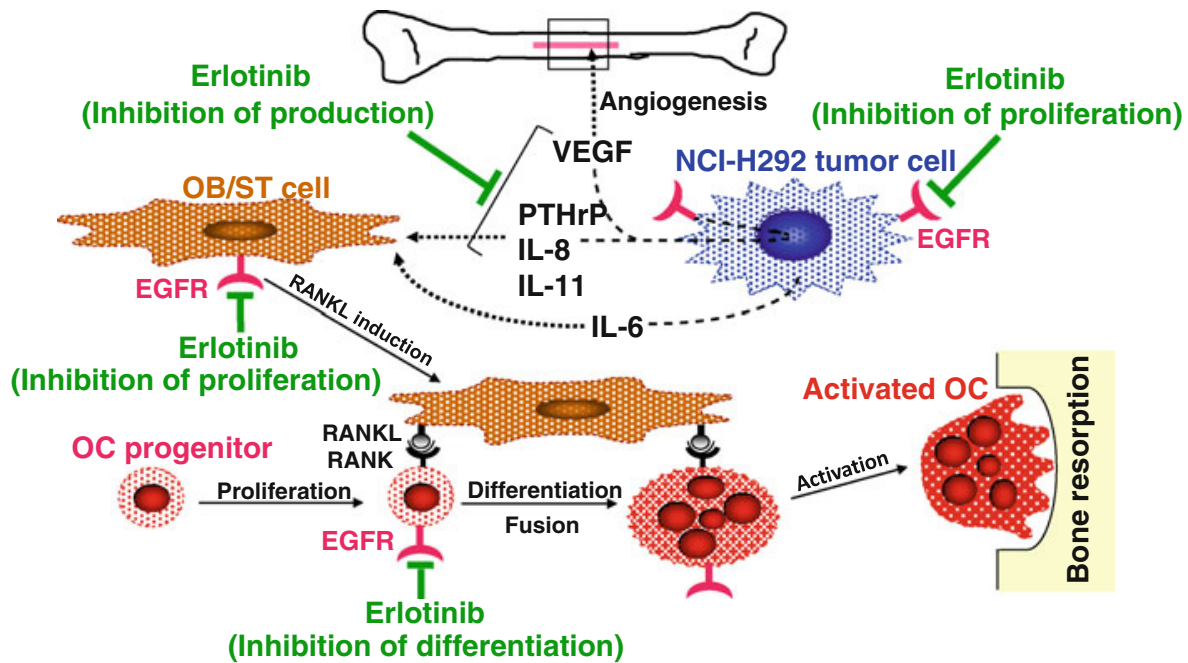

(Fig. 3). The $\mathrm{IC}_{50}$ of NCI-H292 cells with both EGFR and KRAS wild-types was $0.11 \mu \mathrm{M}$. Thus, NCI-H292 cells are considered to be relatively sensitive to erlotinib. These results suggest that erlotinib strongly inhibits the NCIH292 cell proliferation in in vitro by blocking the EGFR signal transduction. In contrast, erlotinib did not inhibit the phosphorylated EGFR expression in tumor cells at the bone metastatic lesions (Fig. 3), which is inconsistent with our in vitro result. It has been reported that the plasma concentration of erlotinib in athymic nude mice correlates well with erlotinib concentration in tumor. It is under $0.0025 \mu \mathrm{M} 24 \mathrm{~h}$ after administration of $100 \mathrm{mg} / \mathrm{kg}$ erlotinib [35]. In this study, the tibiae were collected $24 \mathrm{~h}$ after administration of $75 \mathrm{mg} / \mathrm{kg}$ erlotinib on day 13 . Therefore, the erlotinib concentration in NCI-H292 tumor concentration of erlotinib is considered to under $0.0025 \mu \mathrm{M}$, which is insufficient to block the EGFR phosphorylation. Thus the reduced EGFR phosphorylation in tumor cells may not be detectable even in case of erlotinib treatment.

Second, erlotinib strongly suppressed the production of osteolytic factors, such as PTHrP, IL-8, IL-11 and VEGF in NCI-H292 cells. RANKL expression in osteoblasts/stromal cells is stimulated by various soluble osteolytic factors including PTHrP, IL-6, IL-8, IL-11 which are produced by tumor cells [27]. Especially, PTHrP plays an important role in the development of osteolytic bone metastases in breast cancer [36]. Usually, the addition of osteolytic factors is essential for osteoclast formation in an in vitro coculture system with osteoblasts. In addition, VEGF, a key regulator of angiogenesis and vascular permeability [37], induces the formation of novel vessels in bone metastatic lesions [14]. Our results showed that PTHrP, IL-8, IL-11 and VEGF are produced in NCI-H292 cells and these factors were markedly inhibited by erlotinib (Fig. 4). The mechanism by which EGFR signal activation promotes PTHrP, IL-8, IL-11 and VEGF production in NCI-H292 cells is currently unknown. However, the production of these factors in NCIH292 cells may be regulated by ERK activation, because ERK has been reported to stimulate the gene expression of these factors in culture cells [37-39] and ERK phosphorylation was completely blocked by erlotinib in NCI-H292 cells (Fig. 3). In contrast, the production of IL-6 in NCI$\mathrm{H} 292$ cells was not affected by erlotinib treatment for $24 \mathrm{~h}$. However, erlotinib markedly decreased IL- 6 protein level in conditioned media on day 4 compared to the vehicletreated cells, because the number of NCI-H292 cells was decreased by erlotinib (data not shown). Therefore, IL-6 is also decreased in this model by extended erlotinib treatment, would be involved in suppression of bone metastases of NCI-H292 cells.

Third, erlotinib inhibited the cell proliferation of osteoblasts/stromal cells. Recently, the role of EGFR in osteoblasts and osteoclastogenesis has been focused on $[13,40]$. Several studies have shown the expression of EGFR in bone marrow mesenchymal stem cells (the precursor of osteoblasts), bone marrow stromal cells, osteoblasts and endothelial cells [13, 14, 41]. Furthermore, EGFR ligands have been reported to stimulate the proliferation of these cells [14, 41-43]. In the present study, we showed that mouse as well as human osteoblasts/stromal cells express EGFR and erlotinib inhibited the proliferation of these cells in a dose-dependent manner in vitro (Fig. 5). In contrast, the proliferative activity of osteoblasts/stromal cells in vivo could not be estimated, because there was no detectable Ki-67 immunostaining in osteoblasts/stromal cells. The growth rate of osteoblasts/stromal cells in vivo may be much slower than that of in vitro. Intriguingly, EGFR ligands have been reported to regulate RANKL and OPG expression in osteoblasts/stromal cells [13, 42]. OPG, known as an osteoclastogenesis inhibitory factor, is a decoy receptor of RANK and inhibits osteoclast differentiation by interfering with the RANKL/RANK interaction [44].We 
found that RANKL expression levels in primary osteoblasts decreased about $50 \%$ with erlotinib and there was no change in RANKL expression levels of ST2 cells. In contrast, OPG expression levels of either type of cell were not changed (data not shown).

Fourth, erlotinib completely suppressed the RANKLinduced osteoclast differentiation from mouse bone marrow cells without exhibiting cytotoxicity. Our results are consistent with the previous reports. Wang et al. [24] have shown that EGFR is expressed in osteoclasts and osteoclast recruitment is impaired in EGFR deficient mice. In addition, Yi et al. [40] have demonstrated that EGFR plays a crucial role in RANKL-induced osteoclast differentiation from mouse bone marrow cells by regulating RANKLactivated signaling pathways through cross-talking with RANK. These findings indicate that erlotinib may inhibit the activation of osteoclasts by affecting on non-cancer cells including osteoblasts/stromal cells and osteoclasts, independently by affecting on NCI-H292 tumor cells. Therefore, it is important to highlight the activity of antiEGFR agents on non-cancer cells including osteoblasts/ stromal cells and osteoclasts in the bone metastatic microenvironment. Meanwhile, in clinical, abnormal bone metabolism such as hypercalcemia and bone fracture has not been reported as a side effects of erlotinib, therefore the effects of erlotinib against osteoblasts/stromal cells and osteoclasts may be specific in bone metastatic sites.

Meanwhile, only one cell line, NCI-H292, was used in this study. We have tried to construct another bone metastasis model for generalization of out NCI-H292 model using HCC827 EGFR-mutated NSCLC cell line. Unfortunately, we could not establish HCC 827 bone metastasis model. Therefore, we used only the NCI-H292 osteolytic bone invasion model in the present study. Further studies are needed to clarify whether or not erlotinib could inhibit bone metastases in the other models. Additionally, in this NCI-H292 intra-tibial tumor inoculated osteolytic bone invasion model, it could not be evaluated whether erlotinib have a potential to inhibit forming bone metastasis from primary site through the bloodstream. However, anti-EGFR agents were reported to reduce invasive capacity and angiogenic activity through the inhibition of (1) molecules associated with tissue invasion such as urokinase-type plasminogen activator (UPA) and matrix metalloproteinase (MMP)-9, (2) the proliferation and survival of endothelial cells and (3) the production of VEGF in bone marrow stromal cells (14). Therefore, erlotinib would inhibit the progression of bone metastasis from the primary site through the bloodstream.

Altogether, it is plausible that the inhibition of NCIH292 tumor growth and cell proliferation of osteoblasts/ stromal cells by erlotinib treatment would decrease the chance of interaction between tumor cells and osteoblasts/ stromal cells, resulting in the suppression of the activation of RANKL expression in osteoblasts/stromal cells.

In conclusion, we demonstrated that erlotinib inhibits tumor-induced osteolytic invasion in bone metastasis by suppressing metastatic tumor growth, osteolytic factor production in tumor cells, osteoblasts/stromal cell proliferation and osteoclast differentiation from mouse bone marrow cells. These findings suggest that erlotinib has potential therapeutic benefits against bone metastases of NSCLC and may be a useful therapeutic agent for the treatment of NSCLC patients with bone metastases. Finally, the evidence of our preclinical study would support prospective clinical trials which will clarify the role of erlotinib in the management of NSCLC patients with bone metastases.

Acknowledgments We thank Mr. E. Onuma for special advice. The authors indicated no potential conflicts of interest.

Open Access This article is distributed under the terms of the Creative Commons Attribution Noncommercial License which permits any noncommercial use, distribution, and reproduction in any medium, provided the original author(s) and source are credited.

\section{References}

1. Coleman RE (2001) Metastatic bone disease: clinical features, pathophysiology and treatment strategies. Cancer Treat Rev 27(3):165-176

2. Hirsh VMD, Major PPMDF, Lipton AMD et al (2008) Zoledronic acid and survival in patients with metastatic bone disease from lung cancer and elevated markers of osteoclast activity. J Thorac Oncol 3(3):228-236

3. Dancey J, Sausville EA (2003) Issues and progress with protein kinase inhibitors for cancer treatment. Nat Rev Drug Discov 2(4):296-313

4. Schneider C-PMD, Heigener DMD, Schott-von-Romer KMD et al (2008) Epidermal growth factor receptor-related tumor markers and clinical outcomes with erlotinib in non-small cell lung cancer: an analysis of patients from German centers in the TRUST study. J Thorac Oncol 3(12):1446-1453

5. Pan M, Santamaria M, Wollman DB (2007) CNS response after erlotinib therapy in a patient with metastatic NSCLC with an EGFR mutation. Nat Clin Pract Oncol 4(10):603-607

6. Okano Y, Nishio M (2008) Efficacy of gefitinib in treatment of lung cancer patients with bone metastasis. Clin Calcium 18(4):527-533

7. Sugiura H, Yamada K, Sugiura T et al (2008) Predictors of survival in patients with bone metastasis of lung cancer. Clin Orthop Relat Res 466(3):729-736

8. Zampa G, Moscato M, Brannigan BW et al (2008) Prolonged control of bone metastases in non-small-cell lung cancer patients treated with gefitinib. Lung Cancer 60(3):452-454

9. Satoh H, Ishikawa H, Ohara G et al (2009) Prolonged response to gefitinib in bone metastasis. Med Oncol 26(1):101-102

10. Kim S-J, Uehara H, Karashima T et al (2003) Blockade of epidermal growth factor receptor signaling in tumor cells and tumor-associated endothelial cells for therapy of androgen-independent human prostate cancer growing in the bone of nude mice. Clin Cancer Res 9(3):1200-1210 
11. Weber KL, Doucet M, Price JE et al (2003) Blockade of epidermal growth factor receptor signaling leads to inhibition of renal cell carcinoma growth in the bone of nude mice. Cancer Res 63(11):2940-2947

12. Angelucci A, Gravina GL, Rucci N et al (2006) Suppression of EGF-R signaling reduces the incidence of prostate cancer metastasis in nude mice. Endocr Relat Cancer 13(1):197-210

13. Normanno N, De Luca A, Aldinucci D et al (2005) Gefitinib inhibits the ability of human bone marrow stromal cells to induce osteoclast differentiation: implications for the pathogenesis and treatment of bone metastasis. Endocr Relat Cancer 12(2): $471-482$

14. Normanno N, Gullick WJ (2006) Epidermal growth factor receptor tyrosine kinase inhibitors and bone metastases: different mechanisms of action for a novel therapeutic application? Endocr Relat Cancer 13(1):3-6

15. Feeley BT, Liu NQ, Conduah AH et al (2006) Mixed metastatic lung cancer lesions in bone are inhibited by Noggin overexpression and rank: $\mathrm{Fc}$ administration. J Bone Miner Res 21(10): 1571-1580

16. Sasaki A, Kitamura K, Alcalde RE et al (1998) Effect of a newly developed bisphosphonate, YH529, on osteolytic bone metastases in nude mice. Int J Cancer 77(2):279-285

17. Amizuka N, Shimomura J, Li M et al (2003) Defective bone remodelling in osteoprotegerin-deficient mice. J Electron Microsc (Tokyo) 52(6):503-513

18. Furugaki K, Iwai T, Kondoh K et al (2010) Antitumor activity of erlotinib in combination with gemcitabine in in vitro and in vivo models of KRAS-mutated pancreatic cancers. Oncol Lett 1:231-235

19. Yin JJ, Pollock CB, Kelly K (2005) Mechanisms of cancer metastasis to the bone. Cell Res 15(1):57-62

20. Roodman GD (2007) Bone-breaking cancer treatment. Nat Med 13(1):25-26

21. Guise TA (2009) Breaking down bone: new insight into sitespecific mechanisms of breast cancer osteolysis mediated by metalloproteinases. Genes Dev 23(18):2117-2123

22. Sakai S, Takaishi H, Matsuzaki K et al (2009) 1-Alpha, 25-dihydroxy vitamin D3 inhibits osteoclastogenesis through IFN-beta-dependent NFATc1 suppression. J Bone Miner Metab 27(6):643-652

23. Udagawa N, Takahashi N, Jimi E et al (1999) Osteoblasts/stromal cells stimulate osteoclast activation through expression of osteoclast differentiation factor/RANKL but not macrophage colony-stimulating factor. Bone 25(5):517-523

24. Wang K, Yamamoto H, Chin JR et al (2004) Epidermal growth factor receptor-deficient mice have delayed primary endochondral ossification because of defective osteoclast recruitment. J Biol Chem 279(51):53848-53856

25. Quint LE, Tummala S, Brisson LJ et al (1996) Distribution of distant metastases from newly diagnosed non-small cell lung cancer. Ann Thorac Surg 62(1):246-250

26. Chambers AF, Groom AC, MacDonald IC (2002) Metastasis: dissemination and growth of cancer cells in metastatic sites. Nat Rev Cancer 2(8):563-572

27. Mundy GR (2002) Metastasis: metastasis to bone: causes, consequences and therapeutic opportunities. Nat Rev Cancer 2(8):584-593

28. Yoneda T, Hiraga T (2005) Crosstalk between cancer cells and bone microenvironment in bone metastasis. Biochem Biophys Res Commun 328(3):679-687
29. von Minckwitz G, Jonat W, Fasching P et al (2005) A multicentre phase II study on gefitinib in taxane- and anthracycline-pretreated metastatic breast cancer. Breast Cancer Res Treat 89(2):165-172

30. Marchetti A, Martella C, Felicioni L et al (2005) EGFR mutations in non-small-cell lung cancer: analysis of a large series of cases and development of a rapid and sensitive method for diagnostic screening with potential implications on pharmacologic treatment. J Clin Oncol 23(4):857-865

31. Soung YH, Lee JW, Kim SY et al (2005) Mutational analysis of EGFR and K-RAS genes in lung adenocarcinomas. Virchows Arch 446(5):483-488

32. Kitazawa S, Kitazawa R (2002) RANK ligand is a prerequisite for cancer-associated osteolytic lesions. J Pathol 198(2):228-236

33. Matsuura N, Puzon-McLaughlin W, Irie A et al (1996) Induction of experimental bone metastasis in mice by transfection of integrin alpha 4 beta 1 into tumor cells. Am J Pathol 148(1):55-61

34. Zhao Y, Bachelier R, Treilleux I et al (2007) Tumor $\alpha \mathrm{v} \beta 3$ integrin is a therapeutic target for breast cancer bone metastases. Cancer Res 67(12):5821-5830

35. Higgins B, Kolinsky K, Smith M et al (2004) Antitumor activity of erlotinib (OSI-774, Tarceva) alone or in combination in human non-small cell lung cancer tumor xenograft models. Anti-cancer Drugs 15(5):503-512

36. Bundred NJ, Ratcliffe WA, Walker RA et al (1991) Parathyroid hormone related protein and hypercalcaemia in breast cancer. BMJ 303(6816):1506-1509

37. Bancroft CC, Chen Z, Dong G et al (2001) Coexpression of proangiogenic factors IL- 8 and VEGF by human head and neck squamous cell carcinoma involves coactivation by MEK-MAPK and IKK-NF- $\kappa$ B signal pathways. Clin Cancer Res 7(2):435-442

38. Bamba S, Andoh A, Yasui H et al (2003) Regulation of IL-11 expression in intestinal myofibroblasts: role of c-Jun AP-1- and MAPK-dependent pathways. Am J Physiol Gastrointest Liver Physiol 285(3):G529-G538

39. Lorch G, Gilmore JL, Koltz PF et al (2007) Inhibition of epidermal growth factor receptor signalling reduces hypercalcaemia induced by human lung squamous-cell carcinoma in athymic mice. Br J Cancer 97(2):183-193

40. Yi T, Lee H-L, Cha J-H et al (2008) Epidermal growth factor receptor regulates osteoclast differentiation and survival through cross-talking with RANK signaling. J Cell Physiol 217(2): 409-422

41. Krampera M, Pasini A, Rigo A et al (2005) HB-EGF/HER-1 signaling in bone marrow mesenchymal stem cells: inducing cell expansion and reversibly preventing multilineage differentiation. Blood 106(1):59-66

42. Schneider MR, Sibilia M, Erben RG (2009) The EGFR network in bone biology and pathology. Trends Endocrinol Metab 20(10):517-524

43. Tamama K, Kawasaki H, Wells A (2010) Epidermal growth factor (EGF) treatment on multipotential stromal cells (MSCs). Possible enhancement of therapeutic potential of MSC. J Biomed Biotechnol 2010:795385

44. Lacey DL, Timms E, Tan HL et al (1998) Osteoprotegerin ligand is a cytokine that regulates osteoclast differentiation and activation. Cell 93(2):165-176 